\title{
The correlation between morphological and phylogenetic classification of myxobacteria
}

\author{
Cathrin Spröer, ${ }^{1}$ Hans Reichenbach ${ }^{2}$ and Erko Stackebrandt ${ }^{1}$ \\ Author for correspondence: Erko Stackebrandt. Tel: +495312616 352. Fax: +495312616418. \\ e-mail: erko@dsmz.de
}

DSMZ-Deutsche Sammlung von Mikroorganismen und Zellkulturen $\mathrm{GmbH}^{1}$ and GBF-Gesellschaft für Biotechnologische Forschung $\mathrm{mbH}^{2}, 38124$ Braunschweig, Germany

\begin{abstract}
In order to determine whether morphological criteria are suitable to affiliate myxobacterial strains to species, a phylogenetic analysis of 165 rDNAs was performed on $\mathbf{5 4}$ myxobacterial strains that represented morphologically $\mathbf{2 1}$ species of the genera Angiococcus, Archangium, Chondromyces, Cystobacter, Melittangium, Myxococcus, Polyangium and Stigmatella, five invalid species and three unclassified isolates. The analysis included 12 previously published sequences. The branching pattern confirmed the deep trifurcation of the order Myxococcales. One lineage is defined by the genera Cystobacter, Angiococcus, Archangium, Melittangium, Myxococcus and Stigmatella. The study confirms the genus status of 'Corallococcus', previously 'Chondrococcus', within the family Myxococcaceae. The second lineage contains the genus Chondromyces and the species Polyangium ('Sorangium') cellulosum, while the third lineage is comprised of Nannocystis and a strain identified as Polyangium vitellinum. With the exception of a small number of strains that did not cluster phylogenetically with members of the genus to which they were assigned by morphological criteria ('Polyangium thaxteri' PI t3, Polyangium cellulosum ATCC 25531', Melittangium lichenicola ATCC 25947' and Angiococcus disciformis An d1), the phenotypic classification should provide a sound basis for the description of neotype species in those cases where original strain material is not available or is listed as reference material.
\end{abstract}

Keywords: Myxococcales, 16S rDNA analysis, phylogeny

\section{INTRODUCTION}

The myxobacteria are among the few higher prokaryotic taxa in which morphology and the presence of a complex life cycle of its members can be considered a reflection of their common ancestry. These organisms were first shown to form a phylogenetically coherent cluster by partial 16S rRNA analysis (Ludwig et al., 1983), as a result of which myxobacterial species from five genera formed three major sublines of descent, represented by Nannocystis exedens, 'Sorangium cellulosum' and Myxococcus fulvus and its relatives of the genera Stigmatella and Cystobacter. Later, when a number of $16 \mathrm{~S}$ rDNA oligonucleotide catalogues of hitherto less well-represented genera became available, the myxobacteria, together with the bdellovibrios (Hespell et al., 1984) and the Gram-negative sulfateand sulfur-reducing organisms (Fowler et al., 1986),

The EMBL accession numbers of the 165 rDNA sequences reported in this paper are AJ233897-AJ233950. were described as one of the major eubacterial divisions (Woese et al., 1985). This phylum was subsequently described as the $\delta$-subdivision of the division of purple bacteria (Oyaizu \& Woese, 1985) when its members were found to form an individual branch within the radiation of the division of purple and non-purple bacteria and their relatives, equivalent in phylogenetic depth (i.e. in the range of 16S rRNA identity values separating the most divergent species of this taxon) to the $\alpha$-, $\beta$ - and $\gamma$-subdivisions. This division was later described as the class Proteobacteria (Stackebrandt et al., 1988), with the subdivisions reclassified as subclasses. The most comprehensive phylogenetic analysis of myxobacteria was published by Shimkets \& Woese (1992), who determined the almost complete 16S rRNA sequences of 12 strains belonging to 11 species of nine genera. The results of that study confirmed the partition of species as found in the original study by Ludwig et al. (1983) but broadened the 'Sorangium' (this name has not been validated and 'Sorangium cellulosum' has been reclassified as Polyangium cellulo- 
sum) subline by the inclusion of Chondromyces and Polyangium and the Myxococcus subline by the addition of Angiococcus, Archangium, Melittangium and 'Corallococcus'.

The availability of type strains for species of the order Myxococcales is a point of contention. The Approved Lists of Bacterial Names (Skerman et al., 1980) and subsequent Notification and Validation Lists contain 33 species and nine genera. However, only 12 species of seven genera are available from culture collections. The majority of species (15) are deposited as herbarium material, many of which have lost the ability to grow and to form fruiting bodies, which is the primary basis of species classification. For some species, no type material has been designated at all (e.g. Polyangium aureum, Polyangium luteum, Polyangium parasiticum, Polyangium sorediatum, Polyangium spumosum and Melittangium boletus). The situation is also confused by the fact that several species are listed with a reference strain in the chapter 'Fruiting Gliding Bacteria: The Myxobacteria' in Bergey's Manual of Systematic Bacteriology (McCurdy, 1989), a taxon that has no standing in nomenclature according to Rule 19 of the Bacteriological Code (Lapage et al., 1975). Many type strains are deposited as nonculturable, dried herbarium material (accepted by Rule 18a of the Bacteriological Code), which excludes the possibility of using these strains in comparative phylogenetic and biochemical studies. Morphology and habitats are then the only characteristics that can be used to verify the affiliation of new isolates to known species.

In this study, most of the 54 myxobacterial strains investigated by comparative $16 \mathrm{~S}$ rDNA analysis were tentatively assigned to described species and nonvalidated species on the basis of morphological characteristics. Unfortunately, reference material of many type strains was not available, which limited the present investigation to some extent. In the light of the future effort to replace non-living herbarium material with culturable neotype strains, it was the goal of this study to broaden the phylogenetic basis of species definitions within the order Myxococcales and to test the importance of morphology in the classification of these organisms.

\section{METHODS}

Bacterial strains. Most isolates analysed in this study were new isolates (collection Hans Reichenbach, GBF). All strains were cultivated aerobically on yeast agar (VY/2 agar) plates at $30^{\circ} \mathrm{C}$ (Reichenbach \& Dworkin, 1992). The strains were classified by morphological criteria: shape, size and structure of vegetative cells, myxospores, fruiting bodies and swarm colonies. Myxococcus stipitatus strains were tentatively identified by the bright yellow fluorescence of their swarm colonies under UV irradiation at $366 \mathrm{~nm}$ (Lampky \& Brockman, 1977). Myxococcus xanthus and Myxococcus virescens were distinguished from each other by the greenish-yellow pigmentation of the latter which, however, is easily lost during cultivation. Fruiting-body mor- phology and structure, important characteristics in myxobacterial taxonomy, are often substantially modified or disappear completely after several transfers, which may lead to misidentification.

$16 S$ rDNA sequence determination and analysis. Genomic DNA was extracted from the strains and used for the PCRmediated amplification of the 16S rDNA (Rainey et al., 1996). The purified PCR products were sequenced as described previously (Rainey et al., 1996). The sequences were determined by electrophoresis by using a model $373 \mathrm{~A}$ automatic DNA sequencer (Applied Biosystems).

The phylogenetic position of the myxobacterial strains was determined by using the ae 2 editor (Maidak et al., 1996). Evolutionary distances were calculated by the method of Jukes \& Cantor (1969). Phylogenetic dendrograms were reconstructed by using the neighbour-joining treeing algorithms contained in the PHYLIP package (Felsenstein, 1993) as well as the algorithm of DeSoete (1983). Bootstrap values were determined by using the PHYLIP package (Felsenstein, 1993). The accession numbers of the $16 \mathrm{~S}$ rDNAs of reference strains were: Myxococcus xanthus, M34114; Myxococcus coralloides, M94278; Melittangium lichenicola, M94277; Angiococcus disciformis, M94280; Archangium gephyra, M94273; Cystobacter fuscus, M94276; Stigmatella aurantiaca, M94281; Polyangium ('Sorangium') cellulosum, M94280; Chondromyces crocatus, M94275; Chondromyces apiculatus, M94274; Polyangium sp. P1 4943, M94280; and Nannocystis exedens, M94279.

\section{RESULTS AND DISCUSSION}

The almost complete primary structure of the $16 \mathrm{~S}$ rDNA, consisting of a region between positions 44 and 1465 (Escherichia coli nomenclature; Brosius et al., 1978) was determined for 54 myxobacterial strains isolated at various times from different locations. The $16 \mathrm{~S}$ rDNA sequences have been deposited in the EMBL database under the accession numbers indicated in Table 1 . These sequences, representing 21 species of nine genera and some invalid taxa and nonclassified isolates, were aligned against the 16S rDNA database of 12 myxobacterial strains, including eight type species of seven genera (Oyaizu \& Woese, 1985; Shimkets \& Woese, 1992), and phylogenetic trees were generated. The 16S rDNA of Nannocystis exedens $\mathrm{Na}$ e1, sequenced in this study, was $99.8 \%$ identical to the 16S rRNA sequence of the same strain published by Shimkets \& Woese (1992). The branching patterns of the trees generated were highly similar with the two algorithms used and small differences occurred only in the order of branches among highly related strains. High bootstrap values $(>80 \%)$ supported the trifurcation of the suborders and many other branching points (Fig. 1). The three major lineages were separated from each other by $16 \mathrm{~S}$ rDNA identity values ranging from 73 to $85 \%$.

All strains identified as members of the order Myxococcales on the basis of morphological criteria were also found to be phylogenetically related to members of this order by analysis of their 16S rDNA. The inclusion of the isolates did not change the topology of 
Table 1. Strains investigated in this study, their morphology-based classification and 165 rDNA accession numbers

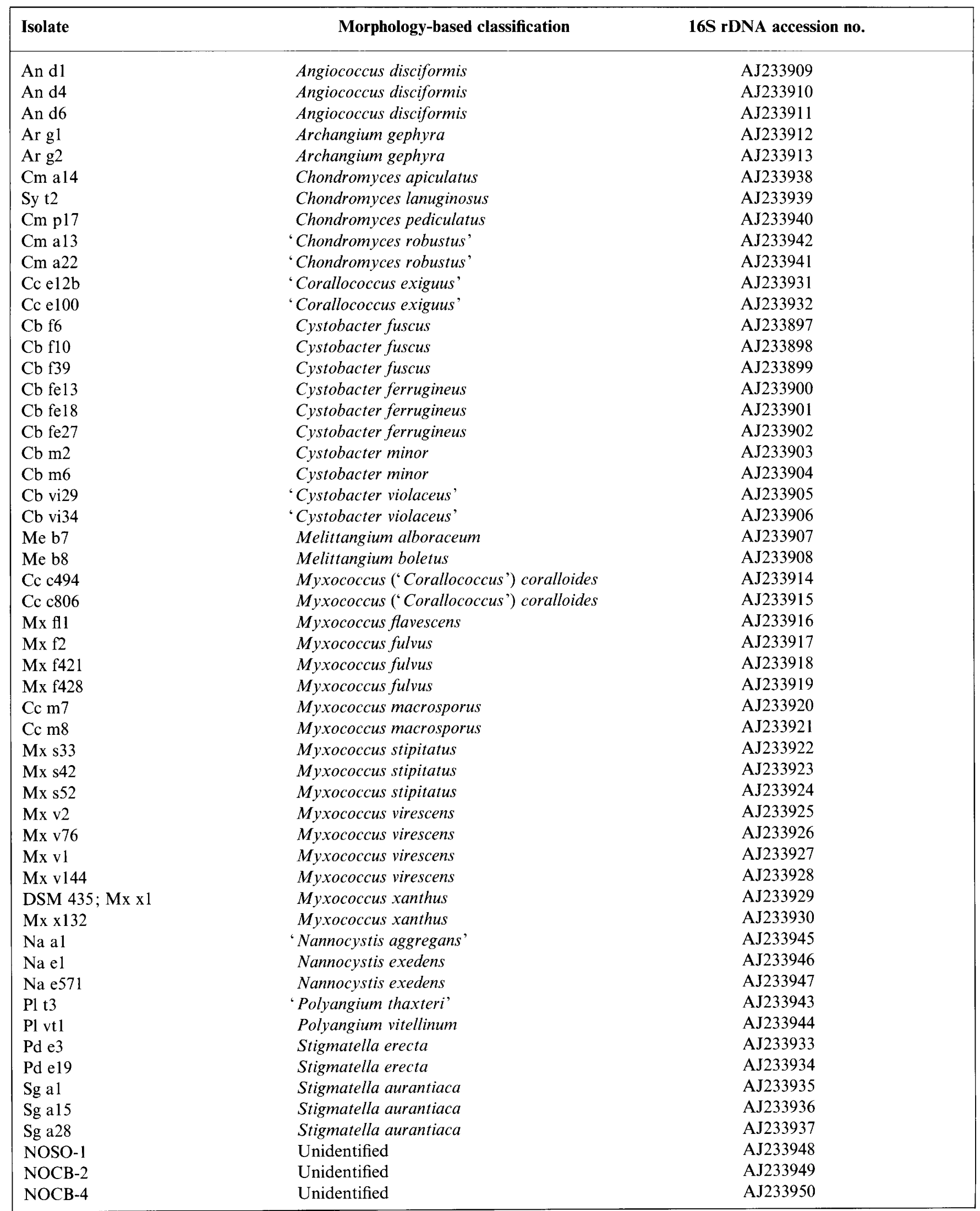




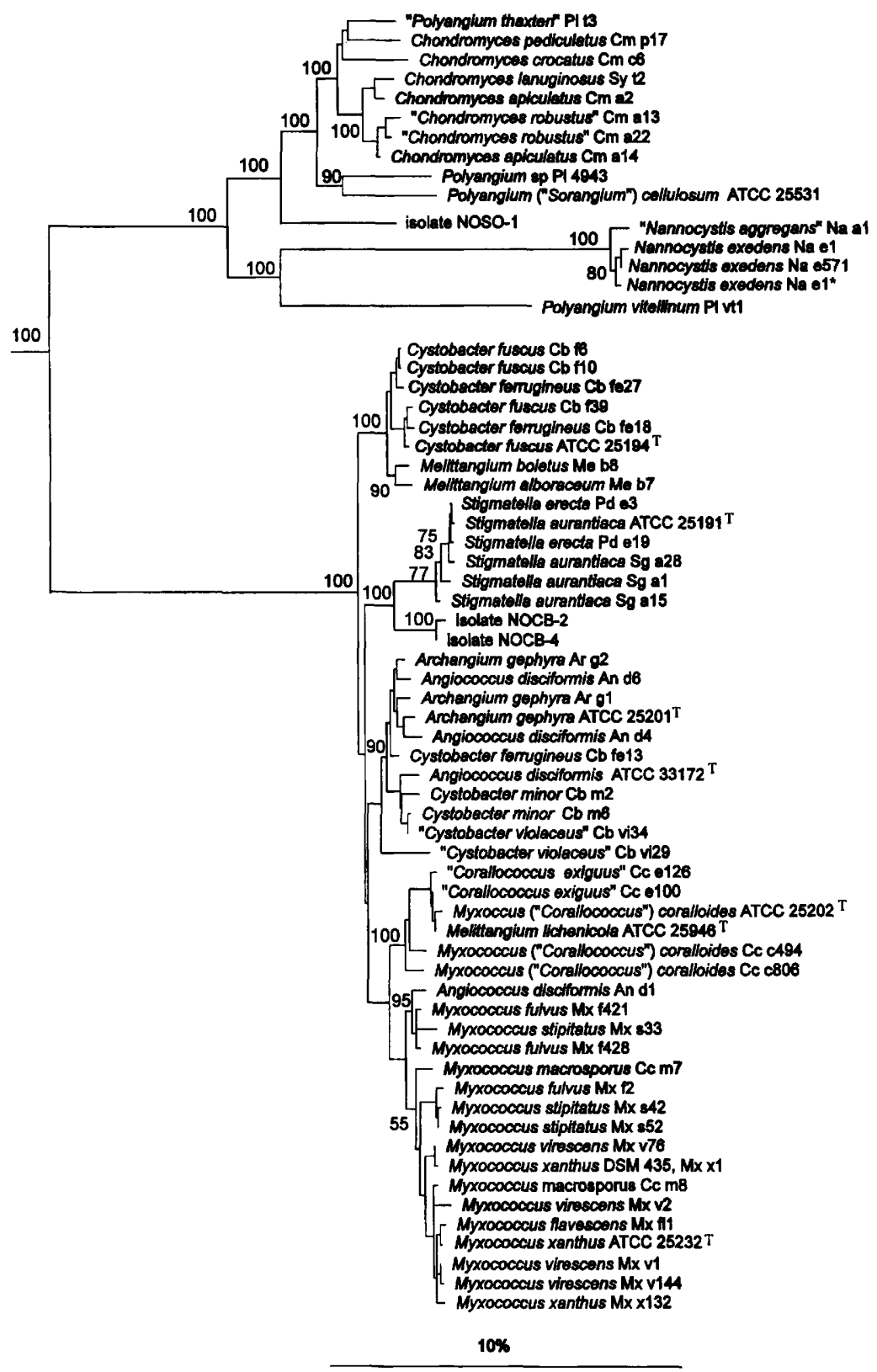

Fig. 1. Neighbour-joining tree of 16S rDNAs showing the phylogenetic position of the type strains of different genera of the order Myxococcales and isolates that were assigned to myxobacterial species on the basis of morphological characteristics (e.g. fruiting bodies, myxospores, colour). The sequences of Gram-negative, sulfate-reducing bacteria were used to root the dendrogram. Numbers within the dendrogram indicate the percentages of occurrence of the branching order in 100 bootstrapped trees. The bar represents 10 nucleotide substitutions per 100 nucleotides.

the phylogenetic tree significantly, with three major branches as originally defined on the basis of the $16 \mathrm{~S}$ rDNA cataloguing approach (Ludwig et al., 1983). Some of the families have been described, i.e. Myxococcaceae Jahn 1924, Polyangiaceae Jahn 1924 emend. Brockman 1989, Archangiaceae Jahn 1924 and Cystobacteraceae McCurdy 1970, while 'Sorangiaceae' has been proposed by Reichenbach \& Dworkin (1992), although the genus name 'Sorangium' has not yet been validated.
The morphology-based affiliation of isolates to genera agreed for most strains, while a higher degree of discrepancies could be observed at the species level. However, for most genera, the intrageneric differences between the $16 \mathrm{~S}$ rDNA primary structures of members were very small, in some cases below $1 \%$. In these cases, the branching pattern was based on sequence differences within highly variable regions of the molecule, which do not necessarily reflect phylogenetic relationships (Stackebrandt \& Goebel, 1994) and 
should not be taken as evidence that species affiliation is erroneous.

\section{Genus Cystobacter}

This genus contains three species, Cystobacter fuscus (the type species), Cystobacter ferrugineus and Cystobacter minor. The epithet minus is incorrect and should be corrected to minor, L. neut. Only reference but no type material is available for these species. While strains of Cystobacter minor were phylogenetically well separated from the other two species and must probably be considered members of a new genus (see below), strains of Cystobacter fuscus and Cystobacter ferrugineus were phylogenetically intermixed at a $16 \mathrm{~S}$ rDNA identity level above $99 \cdot 4 \%$.

\section{Genus Melittangium}

The genus Melittangium is a closely related sister taxon of the genus Cystobacter sensu stricto, from which it was separated by $98.2 \% 16 \mathrm{~S}$ rDNA identity. The branching point was supported by a bootstrap value of $90 \%$. Melittangium contains three species but type material is not available for the type species of the genus, Melittangium boletus. The Melittangium cluster contained two strains, which on the basis of morphological characteristics most closely matched Melittangium boletus (strain $\mathrm{Me}$ b8) and Melittangium alboraceum (Me b7). The type strain of Melittangium lichenicola clustered with strains of 'Corallococcus' and was probably not correctly identified.

\section{Genera Archangium and Angiococcus}

This cluster embraced the type species of Archangium as well as strains assigned to the genera Archangium, Cystobacter and Angiococcus. Closely related to this cluster was a neighbouring taxon, the strains of which are related the neotype strain of Angiococcus disciformis, ATCC $33172^{\mathrm{T}}$. Angiococcus disciformis strain An dl clearly does not belong to the same species as the neotype strain of the species, strain ATCC $33172^{\mathrm{T}}$. The swarm and cell morphology of the latter correspond to those of the genus Cystobacter, to which the species has been transferred in the latest edition of Bergey's Manual of Systematic Bacteriology. Since in our hands the neotype strain no longer produced fruiting bodies and myxospores, comparison of fruiting bodies was not possible. The description of the neotype strain (Hook et al., 1980) suggests that this strain indeed represents the species originally described by Thaxter (1904). The strain was a phylogenetic neighbour of Cystobacter ferrugineus $\mathrm{Cb}$ fe 13 and two moderately related strains of a tentative morphospecies 'Cystobacter violaceus'. Strains of the invalid species 'Cystobacter violaceus' grouped with strains of Cystobacter minor. The Cystobacter minor strains are morphologically similar to Angiococcus disciformis ATCC $33172^{\mathrm{T}}$, supporting the phylogenetic clustering of members of the taxa. The most unrelated strain was 'Cystobacter violaceus' $\mathrm{Cb}$ vi29, showing as little as $97.4 \%$ identity to other members of the cluster.

The phylogenetic separateness of Cystobacter minor and 'Cystobacter violaceus' from the type species, together with unique morphological properties of these strains, supports the proposal to elevate this cluster to genus status.

\section{Genus Myxococcus}

All of the 13 strains identified as members of Myxococcus clustered with the type strain of Myxococcus xanthus, ATCC $19368^{\mathrm{T}}$ (Oyaizu \& Woese, 1985) [listed as ATCC $25232^{\mathrm{T}}$ in the latest edition of the ATCC catalogue of strains (1994)]. The intrageneric structure recognized five subclusters, which were separated by 16S rDNA identity values of about $98.2 \%$ and showed bootstrap values above $55 \%$. The first subcluster contained two isolates identified as members of $M y x \mathrm{x}^{-}$ coccus xanthus, strains $\mathrm{Mx} \times 132$ and Mx x1 (=DSM 435). They shared more than $99 \%$ identity with the type strain, ATCC $25232^{\mathrm{T}}$. Other strains that were highly related to Myxococcus xanthus were identified as Myxococcus virescens (four strains), a strain of Myxococcus flavescens and a single strain of Myxococcus ('Corallococcus') macrosporus (strain Cc m8). The genus name 'Corallococcus' has been proposed by Reichenbach \& Dworkin (1992) for former 'Chondrococcus' species, but neither genus name has been validated so far. The second subcluster consisted of a group of three strains, two of which were identified as Myxococcus stipitatus (Mx s42 and $\mathrm{Mx} \mathrm{s52)}$ and a strain of Myxococcus fulvus (Mx f2). Two strains of Myxococcus fulvus (Mx f421 and Mx f428) and a strain of Myxococcus stipitatus (Mx s33) formed subcluster 3 , while the other two subclusters were defined by single strains of Myxococcus ('Corallococcus') macrosporus $(\mathrm{Cc} \mathrm{m7)}$ and Angiococcus disciformis strain An d1. The data may indicate that the subtle differences in 16S rDNA sequences do not allow a reliable species differentiation. They may also suggest that Myxococcus xanthus and Myxococcus virescens can not be distinguished reliably by pigmentation alone, viz. orange versus greenish-yellow, a colour difference that may disappear during cultivation anyway. DNADNA reassociation should establish whether the two species should be united. Myxococcus flavescens may belong to the same complex. The Myxococcus fulvus and Myxococcus stipitatus strains used in this study appeared to be typical representatives of each species, which are clearly distinguished by smaller, often oval myxospores and the yellow fluorescence mentioned above for Myxococcus stipitatus. Yet, strains of either species were placed at different locations in the dendrogram. The position of the two Myxococcus macrosporus strains is less of a surprise. Both produced only degenerated fruiting bodies and therefore could really represent Myxococcus virescens strains. As both strains have large myxospores, $2-2.5 \mu \mathrm{m}$ in diameter, 
they should cluster together and with Myxococcus virescens. Again, DNA-DNA reassociation studies should resolve the problem of species affiliation. Angiococcus disciformis strain An dl has a typical myxobacterial swarm with soft, snaking veins and spherical myxospores but, in contrast to all other members of the family, produces fruiting bodies with sporangioles. Strain An d1 does not belong to the same species and genus represented by the neotype strain of Angiococcus disciformis, ATCC $33172^{\mathrm{T}}$.

\section{The 'Corallococcus' cluster}

This cluster is a sister taxon of the genus Myxococcus, containing the type strain of Myxococcus ("Corallococcus') coralloides ATCC $25202^{\mathrm{T}}$ (previously 'Chondrococcus') and, unexpectedly, the type strain of Melittangium lichenicola, ATCC $25946^{\mathrm{T}}$. Two strains that were identified as typical members of the emerging genus 'Corallococcus', 'Corallococcus exiguus' Cc e100 and $\mathrm{Cc}$ e216, were also found to be placed within this cluster, while two strains of Myxococcus ('Corallococcus') coralloides, Cc c494 and Cc c806, branched somewhat deeper. The 'Corallococcus' strain cluster was separated from members of Myxococcus by $16 \mathrm{~S}$ rDNA identity values between 97 and $98 \%$. A bootstrap value of $100 \%$ strongly supported the bifurcation point of these two neighbouring taxa. The separate position of 'Corallococcus' species, together with their distinct morphological characteristics and physiological differences, indicate that this taxon merits genus status.

\section{Genus Stigmatella}

This genus, containing two species for which type material is available, formed a phylogenetically welldefined taxon. Strains of the two species showed greater than $99.4 \% 16 \mathrm{~S}$ rDNA sequence identity and the branching pattern of strains did not allow clear discrimination between the two species, Stigmatella erecta and Stigmatella aurantiaca. The two are very difficult to distinguish if fruiting body morphology has somewhat degenerated. A clear difference in genome size appears to support the species differentiation (Neumann et al., 1992).

\section{Suborder Sorangineae}

Genus Chondromyces. The genus Chondromyces consists of five species, all of which have been deposited as herbarium material and hence are not available for phylogenetic analysis. However, Chondromyces strains are the most differentiated morphologically among the myxobacteria, meaning that strains can be allocated to described species with considerable confidence. Comparison of $16 \mathrm{~S}$ rDNA sequences revealed that all isolates affiliated to the species Chondromyces lanuginosus, Chondromyces apiculatus, Chondromyces crocatus and Chondromyces pediculatus formed a phylo- genetically coherent cluster. The phylogenetic distance among certain species, however, indicates that the genus is genomically more diverse than the other genera of the Myxococcales. While some species exhibited 16S rDNA identities above $98.5 \%$ ('Chondromyces robustus', Chondromyces apiculatus and Chondromyces lanuginosus), the species Chondromyces crocatus and Chondromyces pediculatus showed around $96.5 \%$ sequence identity to each other and to other species of the genus. A more detailed analysis of Chondromyces crocatus strains has recently demonstrated the phylogenetic identity of most of the analysed strains. Three strains were slightly less related, showing around $98.5 \% 16 \mathrm{~S}$ rDNA identity to the other strains of the species (Jacobi et al., 1996).

Also included in the Chondromyces cluster is a strain classified as 'Polyangium thaxteri' on the basis of its unique morphology (see Reichenbach \& Dworkin, 1992). This organism produces clusters or chains of large, spherical, orange-brown sporangioles, which may be borne on a short slime pedicle. Thus, the fruiting body has little resemblance to a Chondromyces fruiting body but is also clearly different from those described for Polyangium species.

Genus Polyangium. Ten species of this genus have been described, but with the exception of Polyangium ('Sorangium') cellulosum, culturable reference material is not available for any of them. For many species, not even a reference strain is available. While Polyangium ('Sorangium') cellulosum subsp. 'ferrugineum' ATCC 25531 and Polyangium sp. Pl 4943 (Shimkets \& Woese, 1992) clustered distantly with members of Chondromyces, Polyangium vitellinum $\mathrm{Pl}$ vtl defined a separate line of descent. The morphology of strain Pl vtl most closely matches that of the type species, Polyangium vitellinum, in that the organism produces greenishyellow swarms and large, spherical, yellow sporangioles within and on the substrate. Obviously, the phylogenetic position of Polyangium vitellinum is neither close to Polyangium ('Sorangium') cellulosum ATCC 25531 nor to any species of Chondromyces. Apparently, the genus-specific morphological characteristics of Polyangium do not bear phylogenetic significance, as strains identified as members of this genus were placed at three different positions within the suborder Sorangineae (see below). In fact, the fruiting bodies of the Polyangium species are simply clusters and piles of smaller and larger sporangioles, sometime closely packed and polyhedral, and they resemble the fruiting bodies of Cystobacter and Angiococcus. Cystobacter and Polyangium species have therefore previously been classified together, but can easily be separated by the shape of the vegetative cells. From this it follows that Polyangium cellulosum and possibly 'Polyangium thaxteri' probably represent the nuclei of new genera. The genus name Sorangium should be revived for the cellulose degraders of the Polyangium cellulosum complex. A new genus will be defined for the 'Polyangium' species of the Pl 4943 type, which are very common myxobacteria in soil. 
Genus Nannocystis. The separate position of the genus Nannocystis has already been noted in the first phylogenetic study on myxobacteria (Ludwig et al., 1983) and was later explained by rapidly evolving $16 \mathrm{~S}$ rDNA (Shimkets \& Woese, 1992). The presence of the sequence idiosyncrasies in the strains of Nannocystis investigated in this study was confirmed. The two strains of Nannocystis exedens (Nannocystis exedens $\mathrm{Na}$ el was sequenced twice, see above) shared more than $99.4 \%$ sequence identity, and this species is highly related to the new species 'Nannocystis aggregans' ( $99 \%$ sequence identity).

Unassigned isolates. Three isolates could not be assigned to any of the acknowledged myxobacterial species on the basis of morphological criteria. The phylogenetic analysis confirmed their morphological uniqueness. While isolate NOSO-1, showing some morphological resemblance to species of Polyangium, branched deeply within the suborder Sorangineae, the isolates NOCB-2 and NOCB-4, resembling Cystobacter species, are distantly related to members of Stigmatella. These two new groups of isolates probably represent novel genera.

\section{Conclusions}

The present study confirms not only that the myxobacteria are a phylogenetically coherent group, but, to a large extent, the phylogenetic significance of morphological characteristics of myxobacteria. Strains classified on morphological grounds, such as Archangium gephyra, Cystobacter fuscus, Nannocystis exedens and Stigmatella aurantiaca, indeed clustered next to the respective neotype strains in the phylogenetic analysis. In two cases, no match was observed. The type strain of Angiococcus disciformis, ATCC $33127^{\mathrm{T}}$, clearly does not belong to the family Myxococcaceae. On the basis of cell and swarm morphology, it seems justified that the species has been transferred to the genus Cystobacter. This means that strain An d1, which belongs to the Myxococcaceae, represents a new genus. The second case refers to the neotype strain of Melittangium lichenicola, ATCC $25946^{\mathrm{T}}$, which clustered within the radiation of 'Corallococcus' species and may have been misclassified. There are many fruiting-body-forming strains that conform to the description of the species and those strains certainly do not belong to the family Myxococcaceae.

The main problem identified in this study refers to the availability of type strains for myxobacterial species. The original descriptions of most species are based on fruiting bodies developing in crude cultures and many of these strains have never been cultivated in pure culture by the authors. Consequently, type strains are often only deposited as herbarium specimens that can no longer be cultured. In addition, our experience has shown that herbarium material will not be made available for isolation of DNA, on which 16S rDNA analysis depends. Obviously, the solution is not to rely on herbarium material but to define and deposit neotype strains. As myxobacteria have a highly developed morphology, it should be possible to find strains that correspond to the original descriptions. There is, however, a basic difficulty. Fruiting-body morphology often degenerates or is lost completely during cultivation, with the consequence that the type or neotype strain no longer shows the characteristics of the species. In fact, this observation has made myxobacterial taxonomists hesitate to deposit neotypes. There are, however, two ways to circumvent the problem. Firstly, the use of preservation methods that allow the strains to produce fruiting bodies, e.g. drying fruiting bodies on filter paper at an early stage of cultivation. Secondly, the deposition of type strains together with careful and comprehensive photographic documentation of fruiting body and myxospore morphology, both being made available to interested investigators. Some difficulties will still remain: fruiting bodies often change their shape and structure during cultivation. Thus, members of several genera, e.g. Stigmatella, Cystobacter and Melittangium, may produce Archangium-type fruiting bodies. This may lead to erroneous classification, even by experienced specialists. The search for differentiating physiological properties has not been particularly successful so far. Eventually, systematic monitoring for differentiating characteristics at the chemotaxonomic level, e.g. protein patterns and lipids, and at the molecular level, e.g. species-specific oligonucleotide probes, may help to overcome the presently unsatisfactory taxonomic situation within the Myxococcales.

\section{REFERENCES}

Brosius, J., Palmer, M. L., Kennedy, P. J. \& Noller, H. F. (1978). Complete nucleotide sequence of a 16S ribosomal RNA gene from Escherichia coli. Proc Natl Acad Sci USA 75, 4801-4805.

DeSoete, G. (1983). A least square algorithm for fitting additive trees to proximity data. Psychometrika 48, 621-626.

Felsenstein, J. (1993). PHYLIP (phylogenetic inference package) version 3.5.1. Department of Genetics, University of Washington, Seattle, USA.

Fowler, V. J., Widdel, F., Pfennig, N., Woese, C. R. \& Stackebrandt, E. (1986). Phylogenetic relationship of sulfate- and sulfurreducing eubacteria. Syst Appl Microbiol 6, 32-41.

Hespell, R. B., Paster, J., Macke, T. \& Woese, C. R. (1984). The origin and phylogeny of the bdellovibrios. Syst Appl Microbiol 5, 196-203.

Hook, L. A., Larkin, J. M. \& Brockman, E. R. (1980). Isolation, characterization, and emendation of description of Angiococcus disciformis (Thaxter 1904) Jahn 1924 and proposal of a neotype strain. Int J Syst Bacteriol 30, 135-142.

Jacobi, C. A., Reichenbach, H., Tindall, B. J. \& Stackebrandt, E. (1996). 'Candidatus comitans', a bacterium living in coculture with Chondromyces crocatus (Myxobacteria). Int J Syst Bacteriol 46, 119-122.

Jukes, T. H. \& Cantor, C. R. (1969). Evolution of protein molecules. In Mammalian Protein Metabolism, pp. 21-132. Edited by H. N. Munro. New York: Academic Press.

Lampky, J. R. \& Brockman, E. R. (1977). Fluorescence of Myxococcus stipitatus. Int J Syst Bacteriol 27, 161. 
Lapage, S. P., Sneath, P. H. A., Lessel, E. F., Skerman, V. B. D., Seeliger, H. P. R. \& Clark, W. A. (editors) (1975). International Code of Nomenclature of Bacteria. Washington, DC: American Society for Microbiology.

Ludwig, W., Schleifer, K. H., Reichenbach, H. \& Stackebrandt, E. (1983). A phylogenetic analysis of the myxobacteria Myxococcus fulvus, Stigmatella aurantiaca, Cystobacter fuscus, Sorangium cellulosum and Nannocystis exedens. Arch Microbiol 135, $58-62$.

McCurdy, H. D. (1989). Order Myxococcales Tchan, Pochon and Prévot 1948, 398 ${ }^{A L}$. In Bergey's Manual of Systematic Bacteriology, vol. 3, pp. 2139-2170. Edited by J. T. Staley, M. P. Bryant, N. Pfennig \& J. G. Holt. Baltimore: Williams \& Wilkins.

Maidak, B. L., Olsen, G. J., Larsen, N., Overbeek, R., McCaughey, M. J. \& Woese, C. R. (1996). The Ribosomal Database Project (RDP). Nucleic Acids Res 24, 82-85.

Neumann, B., Pospiech, A. \& Schairer, H. U. (1992). Size and stability of the genomes of the myxobacteria Stigmatella aurantiaca and Stigmatella erecta. J Bacteriol 174, 6307-6310.

Oyaizu, H. \& Woese, C. R. (1985). Phylogenetic relationships among the sulfate respiring bacteria, myxobacteria and purple bacteria. Syst Appl Microbiol 6, 257-263.

Rainey, F. A., Ward-Rainey, N., Kroppenstedt, R. M. \& Stackebrandt, E. (1996). The genus Nocardiopsis represents a phylogenetically coherent taxon and a distinct actinomycete lineage: proposal of Nocardiopsaceae fam. nov. Int J Syst Bacteriol $\mathbf{4 6}$, 1088-1092.

Reichenbach, H. \& Dworkin, M. (1992). The Myxobacteria. In The Prokaryotes. A Handbook on the Biology of Bacteria. Ecophysiology, Isolation, Identification, Applications, pp. 34163487. Edited by A. Balows, G. H. Trüper, M. Dworkin, W. Harder \& K. H. Schleifer. New York: Springer.

Shimkets, L. \& Woese, C. R. (1992). A phylogenetic analysis of the myxobacteria: basis for their classification. Proc Natl Acad Sci USA 89, 9459-9463.

Skerman, V. B. D., McGowan, V. \& Sneath, P. H. A. (1980). Approved lists of bacterial names. Int $J$ Syst Bacteriol 30, 225-420.

Stackebrandt, E. \& Goebel, B. M. (1994). Taxonomic note: a place for DNA-DNA reassociation and $16 \mathrm{~S}$ rRNA sequence analysis in the present species definition in bacteriology. Int $J$ Syst Bacteriol 44, 846-849.

Stackebrandt, E., Murray, R. G. E. \& Trüper, H. G. (1988). Proteobacteria classis nov., a name for the phylogenetic taxon that includes the 'purple bacteria and their relatives'. Int J Syst Bacteriol 38, 321-325.

Thaxter, R. (1904). Notes on the Myxobacteriaceae. Bot Gaz 23, 395-411.

Woese, C. R., Stackebrandt, E., Macke, T. J. \& Fox, G. E. (1985). A phylogenetic definition of the major eubacterial taxa. Syst Appl Microbiol 6, 143-151. 